\title{
EDITORIAL
}

\section{Beating bad bugs}

\author{
The dangers of the weak pipeline of antibiotics have been highlighted for more than a \\ decade, but the near-term prospects for new drugs to combat the pressing public health \\ threat posed by multidrug-resistant bacteria remain bleak. Collaborations like those \\ pioneered for neglected diseases could have a key role in addressing this challenge.
}

In August, a wave of newspaper reports highlighting the threat from a 'superbug' appeared worldwide, based on a study of the prevalence of multidrug-resistant Enterobacteriaceae in India, Pakistan and the $\mathrm{UK}^{1}$. Such stories have become disturbingly common in recent years, but in this case, the concern is heightened by several factors.

First, the Enterobacteriaceae isolates - which posses a gene coding for a metallo- $\beta$-lactamase known as NDM1 that degrades antibiotics incorporating a $\beta$-lactam ring - were resistant to nearly all antibiotic classes tested ${ }^{1}$. Only colistin, a decades-old drug that is not generally used owing to kidney toxicity, and the tetracycline derivative tigecycline (Tygacil; Pfizer), one of the few new antibiotics approved in the past decade, retained activity against most isolates.

Second, the isolates were Gram-negative species such as Klebsiella pneumoniae, rather than the frequently highlighted hospital superbug, methicillin-resistant Staphylococcus aureus (MRSA), which is Gram-positive. Although the late-stage pipeline of drugs active against MRSA is weak, that for key Gram-negative pathogens including K. pneumoniae is non-existent, with no such drugs in Phase III trials and little prospect for novel agents reaching this stage in the near future (see page 675).

Third, most of the isolates carried the NDM1 gene on plasmids that were readily transferable, and many of those from India were from community-acquired infections, suggesting that such plasmids are widespread in the environment. There therefore seems to be substantial potential for NDM1-mediated resistance to rapidly become a global public health problem.

The risks of such problems leading to a catastrophic scenario in which superbugs become sufficiently common that health care, particularly in hospitals, starts to return to something akin to the pre-antibiotic era, have been highlighted for more than a decade. However, although initiatives to combat the spread of antibiotic resistance have been introduced and incentives to stimulate research and development (R\&D) of new antibiotics have been proposed, our ability to effectively combat superbugs is still becoming increasingly compromised.

From a drug discovery perspective, even assuming that commercial incentives sufficient to reverse the exit of much of the pharmaceutical industry from antibacterial $\mathrm{R} \& \mathrm{D}$ around a decade ago can be created, there are still major scientific challenges in the field. An illustration is provided by the experience of GlaxoSmithKline - one of the few large pharmaceutical companies still active in antibacterial R\&D - who pursued an extensive programme between 1995 and 2001 aiming to exploit novel targets emerging from bacterial genomics ${ }^{2}$. Although 70 high-throughput screening campaigns were performed, only 5 leads were identified, well below what was anticipated to be needed to ultimately lead to even one new antibiotic, given that industry data indicated that on average 16 antibiotics entering Phase I trials are required for one to achieve regulatory approval ${ }^{2}$.

Furthermore, the clinical development of antibacterial drugs poses substantial challenges, ranging from the design of non-inferiority trials that are acceptable to regulatory authorities to identifying suitable dosing regimens. Indeed, 4 out of 12 antibiotics reported to be in late-stage development have had their initial application for regulatory approval turned down (see page 676), with regulators requesting additional trials for reasons such as failure to show an acceptable margin of non-inferiority to an active comparator.

The recent report of a new class of antibiotics that inhibit bacterial topoisomerases, which are well-established targets for both Gram-positive and Gram-negative bacteria, is therefore most welcome ${ }^{3}$. Moreover, the model behind this success - which involved collaborations between GlaxoSmithKline, the Seeding Drug Discovery initiative of the UK Wellcome Trust and the US Defense Threat Reduction Agency - is also of substantial interest. As has been shown for diseases such as malaria in the past decade, public-private partnerships can revitalize R\&D in areas in which individual companies are reluctant to engage or are failing to progress in. It is to be strongly hoped that such collaborations will provide a template for accelerating efforts to address the unique and pressing scientific and economic challenges associated with antibiotic R\&D.

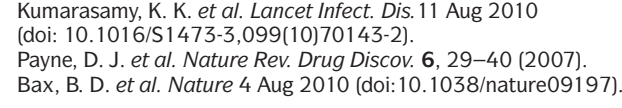

